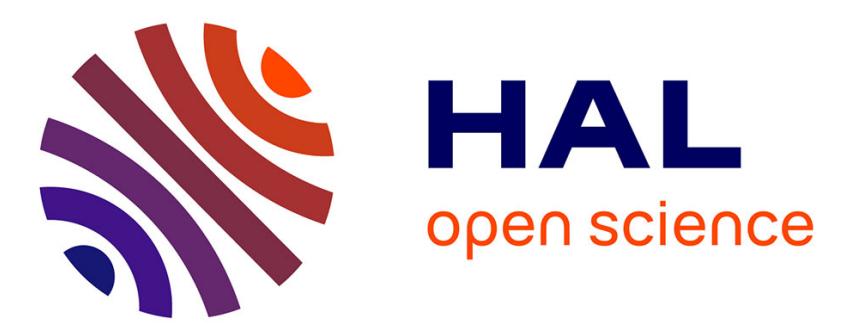

\title{
Rhomboid protein 2 of Eimeria maxima provided partial protection against infection by homologous species
} Yufeng Chen, Di Tian, Lixin Xu, Ruofeng Yan, Xiangrui Li, Muhammad Ali A. Shah, Xiaokai Song

\section{- To cite this version:}

Yufeng Chen, Di Tian, Lixin Xu, Ruofeng Yan, Xiangrui Li, et al.. Rhomboid protein 2 of Eimeria maxima provided partial protection against infection by homologous species. Veterinary Research, 2021, 52 (1), pp.29. 10.1186/s13567-020-00886-7 . hal-03148168

\author{
HAL Id: hal-03148168 \\ https://hal.science/hal-03148168
}

Submitted on 22 Feb 2021

HAL is a multi-disciplinary open access archive for the deposit and dissemination of scientific research documents, whether they are published or not. The documents may come from teaching and research institutions in France or abroad, or from public or private research centers.
L'archive ouverte pluridisciplinaire HAL, est destinée au dépôt et à la diffusion de documents scientifiques de niveau recherche, publiés ou non, émanant des établissements d'enseignement et de recherche français ou étrangers, des laboratoires publics ou privés. 


\title{
Rhomboid protein 2 of Eimeria maxima provided partial protection against infection by homologous species
}

\author{
Yufeng Chen ${ }^{1 \dagger}$, Di Tian ${ }^{1,2 \dagger}$, Lixin Xu ${ }^{1}$, Ruofeng Yan ${ }^{1}$, Xiangrui Li ${ }^{1}$, Muhammad Ali A. Shah ${ }^{3}$ and Xiaokai Song ${ }^{1 *}$ (D)
}

\begin{abstract}
Rhomboid-like proteases (ROMs) are considered as new candidate antigens for developing new-generation vaccines due to their important role involved in the invasion of apicomplexan protozoa. In prior works, we obtained a ROM2 sequence of Eimeria maxima (EmROM2). This study was conducted to evaluate the immunogenicity and protective efficacy of EmROM2 recombinant protein (rEmROM2) and EmROM2 DNA (pVAX1-EmROM2) against infection by Eimeria maxima (E. maxima). Firstly, Western blot assay was conducted to analyze the immunogenicity of rEmROM2. The result showed that $\mathrm{rEmROM} 2$ was recognized by chicken anti-E. maxima serum. Reverse transcription-polymerase chain reaction (RT-PCR) and Western blot assay revealed apparent transcription and expression of EmROM2 at the injection site. qRT-PCR (quantitative real-time PCR), flow cytometry and indirect ELISA indicated that vaccination with rEmROM2 or EmROM2 DNA significantly upregulated the transcription level of cytokines (IFN- $\gamma$, IL-2, IL-4, IL-10, IL-17, TGF- $\beta$ and TNF SF15), the proportion of $\mathrm{CD}^{+}$and $C D 4^{+} T$ lymphocytes and serum IgG antibody response. Ultimately, a vaccination-challenge trial was performed to evaluate the protective efficacy of $r E m R O M 2$ and pVAX1-EmROM2 against E. maxima. The result revealed that vaccination with rEmROM2 or pVAX1-EmROM2 significantly alleviated enteric lesions, weight loss, and reduced oocyst output caused by challenge infection of E. maxima, and provided anticoccidial index (ACI) of more than 160, indicating partial protection against E. maxima. In summary, vaccination with rEmROM2 or pVAX1-EmROM2 activated notable humoral and cell-mediated immunity and provided partial protection against E. maxima. These results demonstrated that EmROM2 protein and DNA are promising vaccine candidates against $E$. maxima infection.
\end{abstract}

Keywords: Rhomboid-like protein 2, Eimeria maxima, Immunogenicity, Protective efficacy

\section{Introduction}

Avian coccidiosis, a kind of intestinal parasitic protozoa disease, seriously impairs the poultry industry worldwide with a loss of beyond $\$ 3$ billion USD per year [1]. Currently, controlling of this disease mainly depends on the usage of anticoccidial drugs worldwide [2,3]. However,

\footnotetext{
*Correspondence: songxiaokai@njau.edu.cn

†Yufeng Chen and Di Tian contributed equally to this work

${ }^{1}$ MOE Joint International Research Laboratory of Animal Health and Food

Safety, College of Veterinary Medicine, Nanjing Agricultural University, Nanjing 210095, China

Full list of author information is available at the end of the article
}

extensive drug use has caused drug resistance and drug residues in food which aroused widespread concern [4, 5]. Live vaccines are the main alternative control strategy to chemical prophylaxis [2]. Nevertheless, the conventional live vaccines are costly, not fully efficient and have a risk of diffusing pathogens $[6,7]$. Vaccination with new generation vaccines including DNA vaccine and subunit vaccine is a promising strategy alternative to conventional treatments $[6,8]$. For developing new generation vaccines, it is important to identify protective antigens. Thus, research efforts have been put in to find novel vaccine targets over the past several decades [9].

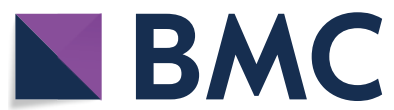

(c) The Author(s) 2021. This article is licensed under a Creative Commons Attribution 4.0 International License, which permits use, sharing, adaptation, distribution and reproduction in any medium or format, as long as you give appropriate credit to the original author(s) and the source, provide a link to the Creative Commons licence, and indicate if changes were made. The images or other third party material in this article are included in the article's Creative Commons licence, unless indicated otherwise in a credit line to the material. If material is not included in the article's Creative Commons licence and your intended use is not permitted by statutory regulation or exceeds the permitted use, you will need to obtain permission directly from the copyright holder. To view a copy of this licence, visit http://creativeco mmons.org/licenses/by/4.0/. The Creative Commons Public Domain Dedication waiver (http://creativecommons.org/publicdomain/ zero/1.0/) applies to the data made available in this article, unless otherwise stated in a credit line to the data. 
A series of specialized parasite molecules are required when apicomplexan protozoa entry into the host cell [10]. Rhomboid-like proteases (ROMs) are involved in the process of invasion by apicomplexan parasites [11]. Invasion by majority of apicomplexan parasites are carried out by adhesins, which mediate binding to the receptor molecules of host cell. ROMs are thought to participate in the cleavage of adhesins from receptors of host cell and result in a final entry into the host cell $[12,13]$. Therefore, ROMs can be regarded as new candidate antigens for developing new-generation vaccines $[13,14]$.

ROMs family have been identified in apicomplexan protozoa, such as Plasmodium spp., Toxoplasma gondii, Theileria annulata and Theileria parva $[13,15,16]$. As for Eimeria species, the protective efficacy of ROMs (such as rFPV-rhomboid, rBCG pMV261-Rho and rBCG pMV361-Rho) from Eimeria tenella (E. tenella) has been evaluated $[9,17,18]$. Up to now, however, there are still few reports concerning the immunogenicity and protective efficacy of E. maxima ROM2.

In prior works, we obtained a ROM2 sequence of $E$. maxima which is the homologous gene with ROM2 of Toxoplasma gondii. In this study, the recombinant protein and eukaryotic expression plasmid of EmROM2 were used as subunit vaccine and DNA vaccine. Meanwhile, the immunogenicity and protective efficacy induced by EmROM2 were evaluated. Our results provide a promising vaccine candidate antigen against E. maxima.

\section{Materials and methods}

\section{Plasmids, parasites, and animals}

The prokaryotic expression vector pET-32a and eukaryotic expression vector pVAX1 were bought from Novagen (Darmstadt, Germany) and Invitrogen (Carlsbad, California, U.S.A), respectively. The oocysts of E. maxima were derived from our laboratory, propagating, harvesting and sporulating were carried out by the method previously described [19]. New-hatched Hy-Line chickens were raised in strictly sterilized animal house. Food and water without anticoccidial drugs were provided ad libitum. SD rats (180-200 g) were purchased from Qinglongshan Breeding Farm in Nanjing. Animal experiments were approved by the Committee on Experimental Animal Welfare and Ethics of Nanjing Agricultural University (Approval number: PAT2020001).

\section{Cloning of EmROM2 and construction of recombinant plasmids of pET-32a-EmROM2 and pVAX1-EmROM2}

Micro glass balls was used to break the sporulated oocysts of E. maxima by whirl mix [19]. Total RNA of E. maxima sporozoites was extracted by an E.Z.N.A. ${ }^{\mathrm{TM}}$ Total RNA Kit I (OMEGA, Norcross, Georgia, U.S.A) following the product instruction. Then, HiScript II Q
RT SuperMix (Vazyme, Nanjing, China) was utilized to generate the cDNAs. RT-PCR was conducted to amplify EmROM2 gene using the specific primers (Table 1). Finally, the PCR products were cloned into prokaryotic expression vector pET-32a and eukaryotic expression vector pVAX1 to create recombinant plasmids pET32a-EmROM2 and pVAX1-EmROM2 respectively. Concisely, EmROM2 gene and pET-32a vector were cleaved by BamH I and Hind III, while EmROM2 gene and pVAX1 vector were cleaved by BamH I and EcoR I, and finally ligated at the same enzyme sites. Double-enzyme digestion and sequencing were conducted to verify the recombinant plasmids.

\section{Preparation of rEmROM2 and anti-rEmROM2 serum}

The expression plasmid of pET-32a-EmROM2 was transformed into the E.coli BL21 (DE3) to express rEmROM2, which was purified using a protein purification kit of His $\operatorname{Trap}^{\mathrm{TM}}$ FF (GE Healthcare, U.S.A). Then a ToxinEraser ${ }^{\mathrm{TM}}$ Endotoxin Removal Kit (Genscript, Nanjing, China) was used to remove the endotoxin to eliminate possible interference. Sodium dodecyl sulfate-polyacrylamide gel electrophoresis (SDS-PAGE) was performed to detect the purified rEmROM2. The rat anti-rEmROM2 serum was prepared by the protocol previously described [8] for Western blot detection. Meanwhile, the serum from noninjected rat was used as a negative control.

\section{Western blot recognition of rEmROM2 by chicken anti-E. maxima serum}

Chicken anti-E. maxima serum was obtained by the method previously described $[8,20]$. The above chicken antiserum was used as primary antibody (serum from uninfected chicken was set as negative control), and horseradish peroxidase (HRP)-conjugated goat antichicken IgG (Sigma-Aldrich, Darmstadt, Germany) was used as secondary antibody to carry out Western blot assay. Briefly, rEmROM2 was separated through SDSPAGE, and next transferred to a nitrocellulose membrane (Merck millipore, Darmstadt, Germany). Subsequently, the membrane was blocked with $5 \%$ bovine serum

Table 1 Primers used for the construction of pET-32aEmROM2 and pVAX1-EmROM2.

\begin{tabular}{ll}
\hline Plasmids & Primers \\
\hline pET-32a-EmROM2 & $\begin{array}{l}\text { Forward: 5'-CGGATCCATGGCGCGGGTTCATACTT-3' } \\
\text { Reverse: 5'-CCAAGCTTTCAGGCGCAACTACGG } \\
\text { GGGAG-3' }\end{array}$ \\
pVAX1-EmROM2 & $\begin{array}{l}\text { Forward: 5'-CGCGGATCCATGGCGCGGGTTCATACTT-3' } \\
\text { Reverse: 5'-CCGGAATTCTCAGGCGCAACTACGGGG } \\
\text { GAG-3' }\end{array}$ \\
\hline
\end{tabular}


albumin (BSA) (Takara Biomedical Technology, Dalian, China) in PBST (phosphate buffered saline-Tween) (20 mM Tris-HCl, $150 \mathrm{mM} \mathrm{NaCl}, 0.05 \%$ (V/V) Tween 20) overnight at $4{ }^{\circ} \mathrm{C}$, afterwards successively incubated with chicken anti-E. maxima serum (1: 100) and goat anti-chicken IgG (1:4500). Finally, 3, 30-diaminobenzidine (DAB) was used to detect the bound antibody [8].

\section{Detection of transcription and expression of the pVAX1-EmROM2 at the injection site through RT-PCR and Western blot}

Fourteen-day-old healthy chickens were divided into two groups at random and vaccinated with $100 \mu \mathrm{g}$ of pVAX1EmROM2 and $100 \mu \mathrm{g}$ of pVAX1 by intramuscular injection of leg, respectively. The pVAX1-injected muscle and non-injected muscle were set as empty vector and negative controls in transcription detection of pVAX1EmROM2. One week later, muscle samples were collected from the pVAX1-EmROM2-injected, pVAX1-injected and non-injected sites. After grinding in a mortar, total RNA of muscle tissue was extracted using RNAiso Plus (Takara Biomedical Technology, Dalian, China) following the product instruction. Then the residual recombinant plasmid was removed by digestion with DNase I (Takara Biomedical Technology, Dalian, China) to eliminate possible interference. RT-PCR was performed to detect the transcription of EmROM2 gene with the RNA product as template using the specific primers for EmROM2 gene (Table 1). In expression detection of pVAX1-EmROM2, RIPA solution (Thermo Scientific, Waltham, MA, U.S.A) was used to treat the pVAX1-EmROM2-injected muscles for $2 \mathrm{~h}$. Then Western blot assay was performed with the supernatant collected. The rat anti-rEmROM2 serum was used as primary antibody (the non-injected rat serum was set as negative control), and HRP-conjugated anti-rat IgG (Sigma-Aldrich, Darmstadt, Germany) was used as secondary antibody to detect the expression of pVAX1EmROM2 at the injected muscle [8].

\section{Immunogenicity analysis of EmROM2 Experimental design}

Fourteen-day-old healthy chickens were divided into six groups (30 chickens per group) at random. In experimental groups, $200 \mu \mathrm{g}$ of rEmROM2 and $100 \mu \mathrm{g}$ of pVAX1EmROM2 were injected into the leg muscles separately. Simultaneously, $200 \mu \mathrm{g}$ of pET-32a tag protein and $100 \mu \mathrm{g}$ of pVAX1 were injected in tag protein control and vector control groups, respectively. Sterile PBS (phosphate buffered saline) was injected in PBS control groups. When the chickens were 21 days old, a booster vaccination was conducted as described above.

\section{Flow cytometry analysis of splenic T lymphocytes subsets}

One week after the primary and booster vaccination, the proportion of $\mathrm{CD}^{+}$and $\mathrm{CD} 4^{+} \mathrm{T}$ lymphocytes was detected with the spleens of five chickens from each group. Splenic lymphocytes were collected according to the previous protocol [21]. Under dark conditions, lymphocytes $\left(1 \times 10^{7}\right.$ cells $\left./ \mathrm{mL}\right)$ were incubated with mouse anti-chicken $\mathrm{CD}^{+}$and mouse anti-chicken $\mathrm{CD}^{+}$, mouse anti-chicken $\mathrm{CD}^{+}$and mouse anti-chicken $\mathrm{CD} 4^{+}$ (SouthernBiotech, Birmingham, AL, U.S.A) for $45 \mathrm{~min}$ at $4{ }^{\circ} \mathrm{C}$, respectively. Then PBS was used to wash the cells twice by centrifugation $\left(2500 \mathrm{rpm}, 3 \mathrm{~min}, 4{ }^{\circ} \mathrm{C}\right)$. T lymphocytes subsets analysis was conducted using BD FACScan flow cytometer (BD Biosciences, Franklin Lakes, NJ, U.S.A).

\section{Detection of cytokines transcription through quantitative real-time $P C R$}

Total RNA of splenic lymphocytes was extracted by an E.Z.N.A. ${ }^{\mathrm{TM}}$ Total RNA Kit I (OMEGA, Norcross, Georgia, U.S.A) following the product instruction. Subsequently, cDNAs of lymphocytes were generated using HiScript II Q RT SuperMix (Vazyme, Nanjing, China) for qRT-PCR assay following the manufacturer's instruction. Specific primers for cytokines of IFN- $\gamma$, IL-2, IL-4, IL-10, IL-17, TGF- $\beta$ and TNF SF15 were designed by NCBI and Primer Ques Tool (IDT). GAPDH gene was designed as internal reference control (Table 2). Meanwhile, amplification efficiencies were evaluated according to the protocol previously reported [22]. qRT-PCR was employed to determine the above mRNA level according to the instruction of ChamQ ${ }^{\mathrm{TM}}$ SYBR qPCR Master Mix Kit (Vazyme, Nanjing, China). Determination of cytokines transcription was performed using the 7500 Real Time PCR System (Applied Biosystems, Carlsbad, CA, U.S.A) with a particular program in accordance with the instruction. The fold change of the transcriptional level of cytokines was determined utilizing $2^{-\Delta \Delta C T}$ method compared to the internal reference control gene of GAPDH [23].

\section{Detection of serum IgG antibody level}

Blood samples were gathered from the chickens 1 week after the primary and booster vaccination. Serum IgG antibody level was determined using indirect ELISA (enzyme-linked immunosorbant assay) [20]. In brief, $0.05 \mathrm{M}$ carbonate buffer was used to dilute the purified rEmROM2 with a concentration of $10 \mathrm{ng} / \mu \mathrm{L}$. A 96-well microtiter plate (Corning-Costar NY, U.S.A) was coated with $150 \mu \mathrm{L}$ of above recombinant protein per well overnight at $4{ }^{\circ} \mathrm{C}$. Subsequently, the plate was blocked with $4.5 \%$ skimmed milk in PBST, and successively incubated with the chicken serum sample $(1: 100)$ and 
Table 2 Primers used for the quantitative real-time PCR.

\begin{tabular}{|c|c|c|c|c|}
\hline RNA target & Primers sequence & Accession NO & $\begin{array}{l}\text { Amplification } \\
\text { efficiency }(\%)^{a}\end{array}$ & $\begin{array}{l}\text { Correlation } \\
\text { coefficients } \\
\left(r^{2}\right)\end{array}$ \\
\hline \multirow[t]{2}{*}{ GAPDH } & Forward: 5'-GGTGGTGCTAAGCGTGTTAT-3' & K01458 & 100.74 & 0.9917 \\
\hline & Reverse: 5'-ACCTCTGTCATCTCTCCACA-3' & & & \\
\hline \multirow[t]{2}{*}{ IL-2 } & Forward: 5'-TAACTGGGACACTGCCATGA-3' & AF000631 & 102.44 & 0.9921 \\
\hline & Reverse: 5'-GATGATAGAGATGCTCCATAAGCTG-3' & & & \\
\hline \multirow[t]{2}{*}{ IL-4 } & Forward: 5'-ACCCAGGGCATCCAGAAG-3' & AJ621735 & 99.09 & 0.9936 \\
\hline & Reverse: 5'-CAGTGCCGGCAAGAAGTT-3' & & & \\
\hline \multirow[t]{2}{*}{ IL-10 } & Forward: 5'-GGAGCTGAGGGTGAAGTTTGA-3' & AJ621614 & 99.19 & 0.9923 \\
\hline & Reverse: 5'-GAAGCGCAGCATCTCTGACA-3' & & & \\
\hline \multirow[t]{2}{*}{ IL-17 } & Forward: 5'-ACCTTCCCATGTGCAGAAAT-3' & EF570583 & 100.24 & 0.9940 \\
\hline & Reverse: 5'-GAGAACTGCCTTGCCTAACA-3' & & & \\
\hline \multirow[t]{2}{*}{ IFN- $\gamma$} & Forward: 5'-AGCTGACGGTGGACCTATTATT-3' & Y07922 & 103.07 & 0.9868 \\
\hline & Reverse: 5'-GGCTTTGCGCTGGATTC-3' & & & \\
\hline \multirow[t]{2}{*}{ TGF- $\beta$} & Forward: 5'-CGGGACGGATGAGAAGAAC-3' & M31160 & 102.79 & 0.9815 \\
\hline & Reverse: 5'-CGGCCCACGTAGTAAATGAT-3' & & & \\
\hline \multirow[t]{2}{*}{ TNF SF15 } & Forward: 5'-GCTTGGCCTTTACCAAGAAC-3' & NM_001024578 & 100.57 & 0.9930 \\
\hline & Reverse: 5'-GGAAAGTGACCTGAGCATAGA-3' & & & \\
\hline
\end{tabular}

amplification efficiency $(\%)=\left(10^{-1 / \text { slope }}-1\right) \times 100 \%$.

goat anti-chicken IgG antibody (Sigma-Aldrich, Darmstadt, Germany) (1:4500). Each well was incubated with 3,3,5,5-tetramethylbenzidine (TMB) (Sigma-Aldrich, Darmstadt, Germany) to develop the color. $2 \mathrm{M} \mathrm{H}_{2} \mathrm{SO}_{4}$ was used to stop the reaction. Finally, a microplate reader (Thermo Scientific, Waltham, MA, U.S.A) was utilized to detect the absorbance.

\section{Protective efficacy evaluation of EmROM2} against homologous infection in chickens

As shown in Table 3, 14-day-old chickens were weighed and divided into eight groups (30 chickens per group) at random. The experimental groups were intramuscularly vaccinated with $200 \mu \mathrm{g}$ of rEmROM2 and $100 \mu \mathrm{g}$ of pVAX1-EmROM2, respectively. Meanwhile, the tag protein control and vector control groups were injected with $200 \mu \mathrm{g}$ of pET-32a tag protein and $100 \mu \mathrm{g}$ of pVAX1 plasmid as the same method as the experimental group, respectively. The challenged control groups and unchallenged control groups were injected with $200 \mu \mathrm{L}$ of sterile PBS. When the chickens were 21 days old, a booster vaccination was conducted as described above. At the age of 28 days, all the chickens, with the exception of the unchallenged control groups, were orally challenged with the sporulated oocysts of $E$. maxima $\left(1 \times 10^{5} /\right.$ chicken $)$ [24]. One week later, weighting and slaughtering the

Table 3 Protective efficacy of rEmROM2 and pVAX1-EmROM2 against challenge with E. maxima.

\begin{tabular}{|c|c|c|c|c|c|c|}
\hline Groups & $\begin{array}{l}\text { Average body } \\
\text { weight gain (g) }\end{array}$ & $\begin{array}{l}\text { Relative body } \\
\text { weight gain (\%) }\end{array}$ & Mean lesion scores & Average OPG $\left(\times 10^{5}\right)$ & $\begin{array}{l}\text { Oocyst } \\
\text { decrease ratio } \\
(\%)\end{array}$ & $\mathrm{ACl}$ \\
\hline rEmROM2 & $50.25 \pm 6.33^{b}$ & $88.30^{b}$ & $1.67 \pm 0.65^{b}$ & $0.51 \pm 0.68^{b}$ & $73.33^{b}$ & 170.60 \\
\hline pET-32a control & $29.46 \pm 11.25^{c}$ & $51.77^{c}$ & $2.66 \pm 0.93^{c}$ & $2.15 \pm 0.97^{c}$ & $4.44^{c}$ & 85.17 \\
\hline Challenged control & $27.21 \pm 8.52^{c}$ & $47.81^{c}$ & $2.84 \pm 0.88^{c}$ & $2.25 \pm 0.94^{c}$ & $0^{c}$ & 79.41 \\
\hline Unchallenged control & $56.91 \pm 10.24^{\mathrm{a}}$ & $100^{\mathrm{a}}$ & $0 \pm 0^{\mathrm{a}}$ & $0 \pm 0^{\mathrm{a}}$ & $100^{\mathrm{a}}$ & 200 \\
\hline pVAX1-EmROM2 & $63.84 \pm 8.80^{b}$ & $80.54^{b}$ & $1.68 \pm 0.99^{b}$ & $0.57 \pm 0.31^{b}$ & $79.72^{b}$ & 162.74 \\
\hline pVAX1 control & $38.19 \pm 15.39^{c}$ & $48.15^{c}$ & $2.75 \pm 0.62^{c}$ & $2.80 \pm 0.16^{c}$ & $0.36^{c}$ & 80.65 \\
\hline Challenged control & $39.28 \pm 9.72^{c}$ & $49.53^{c}$ & $2.83 \pm 0.72^{c}$ & $2.81 \pm 0.13^{c}$ & $0^{c}$ & 81.23 \\
\hline Unchallenged control & $79.32 \pm 9.59^{a}$ & $100^{\mathrm{a}}$ & $0 \pm 0^{\mathrm{a}}$ & $0 \pm 0^{\mathrm{a}}$ & $100^{\mathrm{a}}$ & 200 \\
\hline
\end{tabular}

Significant difference $(p<0.05)$ between data was annotated with different letters in the same column. No significant difference $(p>0.05)$ between data was annotated with the same letter in the same column. 
chickens were performed. Finally, the protective efficacy of rEmROM2 and pVAX1-EmROM2 was evaluated based on the average body weight gain, oocyst output, enteric lesion and ACI (anti-coccidial index) [25-28].

\section{Statistical analysis}

One-way analysis of variance (ANOVA) was used to analyze the data obtained in accordance with Duncan's multiple range test at a 5\% level by using SPSS 20 Data Editor (SPSS Inc., Chicago, IL, U.S.A).

\section{Results}

\section{Cloning of EmROM2 and construction of recombinant plasmids of pET-32a-EmROM2 and pVAX1-EmROM2}

PCR product of EmROM2 gene was detected by electrophoresis and sequencing analysis. As shown in Figure 1, electrophoresis revealed a band of $849 \mathrm{bp}$, which is equal to the target gene. Sequencing analysis revealed that the EmROM2 gene shared 100\% similarity in nucleotide sequence with the gene in GenBank (Sequence ID: XM_013480878.1). Endonuclease digestion and sequencing were performed to verify the constructed recombinant plasmids of pET-32a-EmROM2 and pVAX1-EmROM2. Digestion of pET-32a-EmROM2 with BamH I and Hind III generated a band about $849 \mathrm{bp}$, which is equal to EmROM2 gene, and a larger band of pET-32a vector (Figure 1B). After digestion with $B a m \mathrm{H}$ I and EcoR I, pVAX1-EmROM2 generated a band about 849 bp of the EmROM2 gene, and a larger band, which is the linearized pVAX1 vector (Figure 1D). The sequencing analysis also verified the recombinant plasmids.

\section{Expression of rEmROM2 and Western blot recognition of rEmROM2 by chicken anti-E. maxima serum}

SDS-PAGE revealed that $\mathrm{rEmROM} 2$ was expressed and well purified, showing a band of approximately $49 \mathrm{kDa}$ which is in accordance with the predicted molecular consist of EmROM2 and pET-32a tag protein (Figure 2A, lane 4). SDS-PAGE analysis on the expression of pET32a-EmROM2 in different time was showed in Additional file 1. Western blot assay indicated that rEmROM2 was identified by chicken anti-E. maxima serum (Figure $2 \mathrm{~B}$, lane 1 ).

\section{Transcription and expression detection of recombinant plasmid pVAX1-EmROM2 at the injection site} The transcription of pVAX1-EmROM2 in the injected muscles was detected through RT-PCR. As shown in Figure 3 , a band about 849 bp was detected from the muscles injected with pVAX1-EmROM2 by agarose electrophoresis (Figure 3A, lane 1), indicating the transcription of pVAX1-EmROM2 at the injected muscle. Meanwhile, no

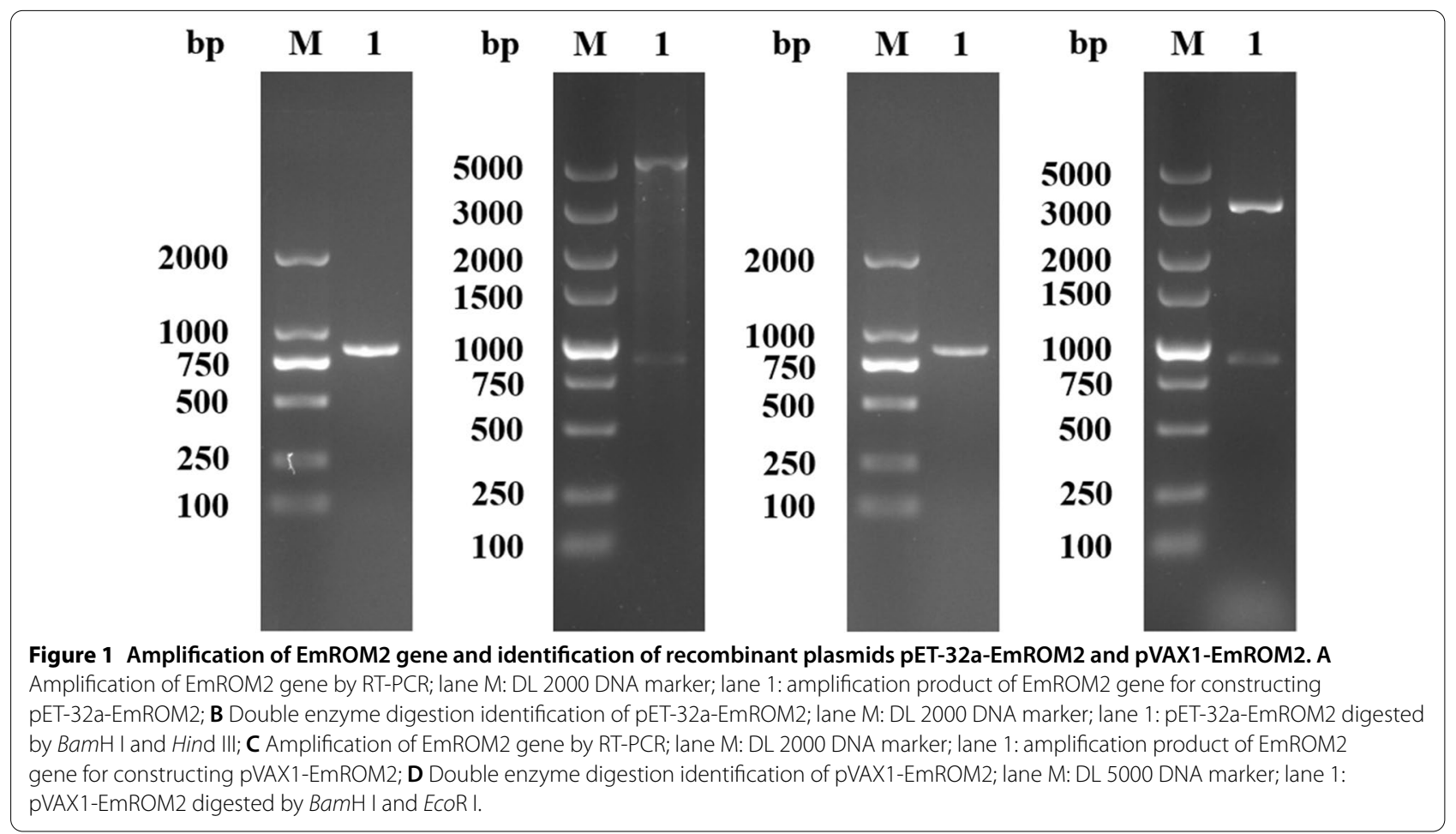




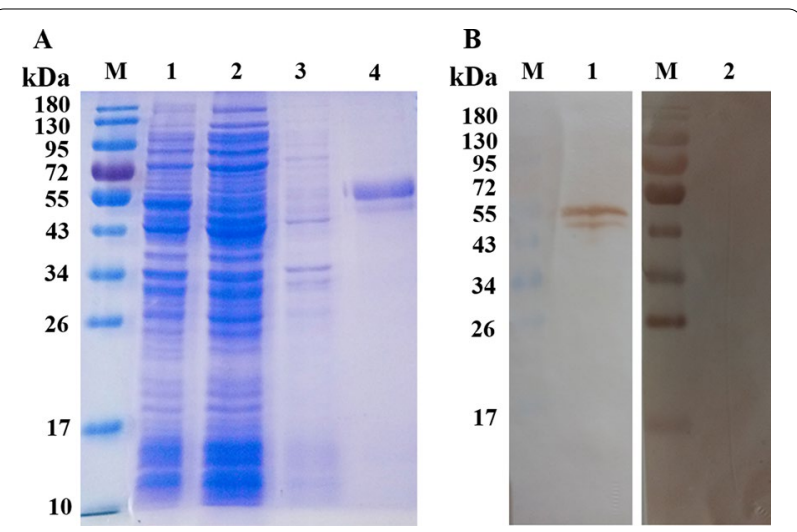

Figure 2 Purification of $r E m R O M 2$ and Western blot recognition of rEmROM2 by chicken anti-E. maxima serum. A Purification of rEmROM2; lane M: standard protein molecular weight marker; lane 1: pET-32a-EmROM2 bacterial lysate; lane 2: the supernatant of pET-32a-EmROM2 bacterial lysate; lane 3: the precipitation of pET-32a-EmROM2 bacterial lysate; lane 4: purified rEmROM2; B Western blot recognition of $\mathrm{rEmROM} 2$ by chicken anti-E. maxima serum; lane M: standard protein molecular weight marker; lane 1: rEmROM2 recognized by chicken anti-E. maxima serum; lane 2: rEmROM2 recognized by negative chicken serum.

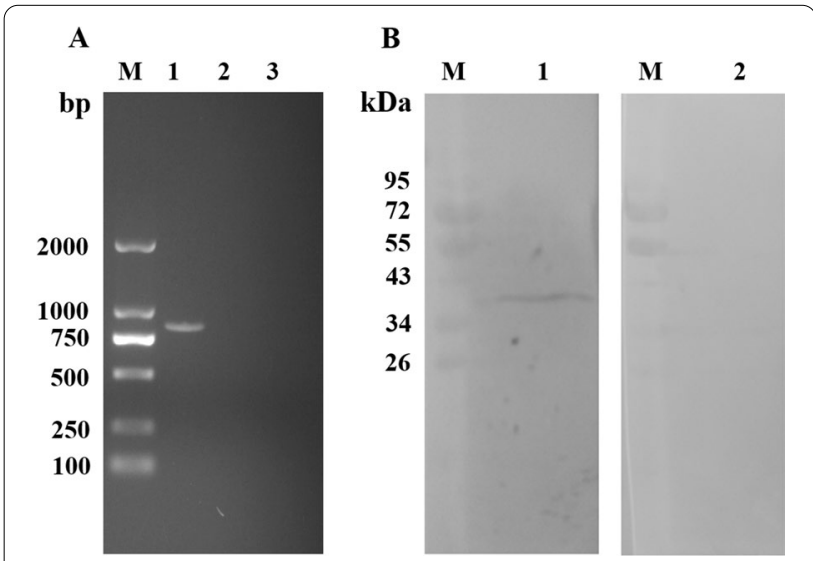

Figure 3 Transcription and expression detection of recombinant plasmid pVAX1-EmROM2 at the injection site. A Transcription detection of pVAX1-EmROM2 by RT-PCR; lane M: DL 2000 DNA marker; lane 1: product of EmROM2 from pVAX1-EmROM2-injected muscle; lane 2: pVAX1-injected muscle control; lane 3: non-injected muscle control; $\mathbf{B}$ Expression detection of pVAX1-EmROM2 by Western blot; lane M: standard protein molecular weight marker; lane 1: pVAX1-EmROM2-injected muscle recognized by rat anti-rEmROM2 serum; lane 2: pVAX1-EmROM2-injected muscle recognized by negative rat serum.

band was found from non-injected muscle and pVAX1injected muscle (Figure 3A, lanes 2 and 3). Western blot assay was conducted to detect the expression of pVAX1EmROM2 from the injected muscles. The result revealed a single reaction band in the pVAX1-EmROM2 injected group (Figure 3B, lane 1), and no band was found in the negative control group (Figure 3B, lane 2).

\section{Immunogenicity analysis of EmROM2 Changes of T lymphocytes subsets induced by rEmROM2 and pVAX1-EmROM2}

Flow cytometry was performed to analyze the changes in the proportion of $\mathrm{CD}^{+} / \mathrm{CD}^{+}$and $\mathrm{CD}^{+} / \mathrm{CD}^{+} \mathrm{T}$ lymphocytes from the EmROM2-vaccinated chickens. The results illustrated that, by comparison with control groups, vaccination with rEmROM2 (Figure 4) or pVAX1-EmROM2 (Figure 5) obviously upregulated the proportion of $\mathrm{CD}^{+}$and $\mathrm{CD} 4^{+} \mathrm{T}$ lymphocytes 1 week after the primary and booster vaccination $(p<0.05)$. No notable differences were found between the pET-32a tag protein control and PBS control $(p>0.05)$, along with the pVAX1 control and PBS control $(p>0.05)$.

\section{Changes of cytokines in transcriptional level induced by $r E m R O M 2$ and $p V A X 1-E m R O M 2$}

qRT-PCR was conducted to detect the changes in transcriptional level of IFN- $\gamma$, IL-2, IL-4, IL-10, IL-17, TGF$\beta 4$ and TNF SF15 cytokines. The rEmROM2-indcued changes in cytokines were shown in Figure 6. One week after the primary vaccination, the transcriptional level of IFN- $\gamma$, IL-2, IL-4, IL-10, TGF- $\beta 4$ and TNF SF15 was obviously upregulated $(p<0.05)$, while compared to the control groups, no notable difference was found in the transcriptional level of IL-17 cytokine $(p>0.05)$. However, by comparison with the control groups, the transcriptional level of all cytokines detected was significantly increased 1 week after the booster vaccination $(p<0.05)$. No notable differences were found between the pET-32a tag protein control group and PBS control group $(p>0.05)$. In the pVAX1-EmROM2 vaccinated group (Figure 7), compared to the control groups, vaccination significantly increased the transcriptional level of all the cytokines detected 1 week after the primary and booster vaccination $(p<0.05)$. Meanwhile, no notable differences were found between the PVAX1 control group and PBS control group $(p>0.05)$.

\section{Changes of serum IgG antibody level induced by rEmROM2 and pVAX1-EmROM2}

Specific serum IgG antibody level from vaccinated chickens was detected using indirect ELISA. In the rEmROM2-vaccinated group (Figure 8) and pVAX1EmROM2 vaccinated group (Figure 9), by comparison with the control groups, vaccination significantly increased the serum IgG level from the vaccinated chickens 1 week after the primary and booster vaccination 
A

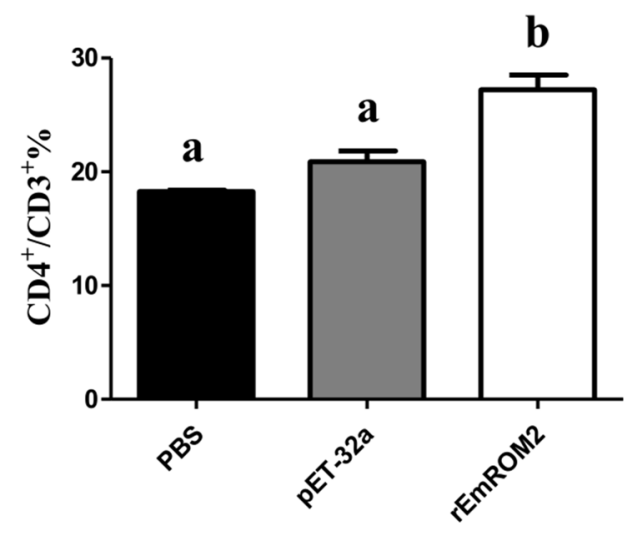

C

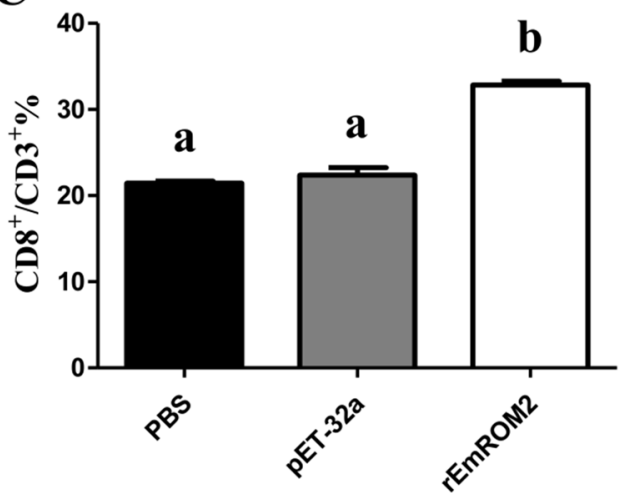

B

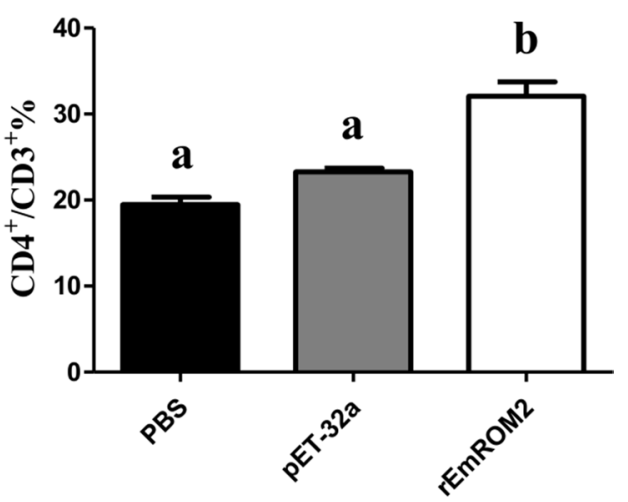

D

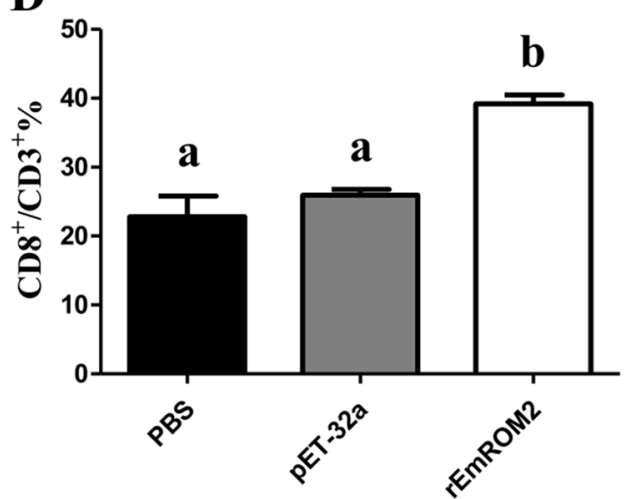

Figure 4 Changes of splenic T lymphocytes subsets induced by rEmROM2 in chickens. A Proportion of $\mathrm{CD}^{+} / \mathrm{CD}^{+}$splenic $\mathrm{T}$ lymphocytes in chickens 1 week after the primary vaccination; $\mathbf{B}$ Proportion of $\mathrm{CD}^{+} / \mathrm{CD}^{+}$splenic $\mathrm{T}$ lymphocytes in chickens 1 week after the booster vaccination; C Proportion of $\mathrm{CD}^{+} / \mathrm{CD}^{+}$splenic T lymphocytes in chickens 1 week after the primary vaccination; $\mathbf{D}$ Proportion of $\mathrm{CD}^{+} / \mathrm{CD}^{+}$splenic $\mathrm{T}$ lymphocytes in chickens 1 week after the booster vaccination. Significant difference $(p<0.05)$ between different groups was annotated with different letters. No significant difference $(p>0.05)$ between different groups was annotated with the same letter.

$(p<0.05)$. No notable differences were found between the pET-32a tag protein control and PBS control $(p>0.05)$, along with the PVAX1 control and PBS control $(p>0.05)$.

\section{Protective efficacy of rEmROM2 and pVAX1-EmROM2 against $E$. maxima infection}

Protective efficacy of rEmROM2 and pVAX1-EmROM2 was evaluated via challenge with E. maxima (Table 3). Chickens vaccinated with rEmROM2 or pVAX1EmROM2 significantly alleviated intestinal lesions, weight loss, and reduced oocyst output compared to chickens in challenged and $\mathrm{pET}-32 \mathrm{a}$ tag protein control groups $(p<0.05)$, as well as the chickens in challenged and pVAX1 control groups $(p<0.05)$. No significant differences were observed between the challenged control and pET-32a tag protein control groups $(p>0.05)$, along with the challenged control and pVAX1 control groups $(p>0.05)$. Moreover, ACI values of rEmROM2-vaccinated group and pVAX1-EmROM2-vaccinated group were 170.60 and 162.74 respectively, indicating partial protection against $E$. maxima infection.

\section{Discussion}

As a serious intestinal disease, avian coccidiosis causes severe economic losses to the poultry industry worldwide. Vaccination with subunit or DNA vaccines is a promising alternative strategy of disease control compared to chemical prophylaxis and vaccination with live vaccines [6, 8]. In recent years, couples of antigens of $E$. maxima were tested as candidate antigens for subunit or DNA vaccines and showed promising protective efficacy, such as gam56 and gam82 [29], EmMIC2 and EmMIC7 $[30,31]$ and some Eimeria common antigens (e.g. GAPDH and 14-3-3) [8, 32]. Since ROMs are involved in 
A

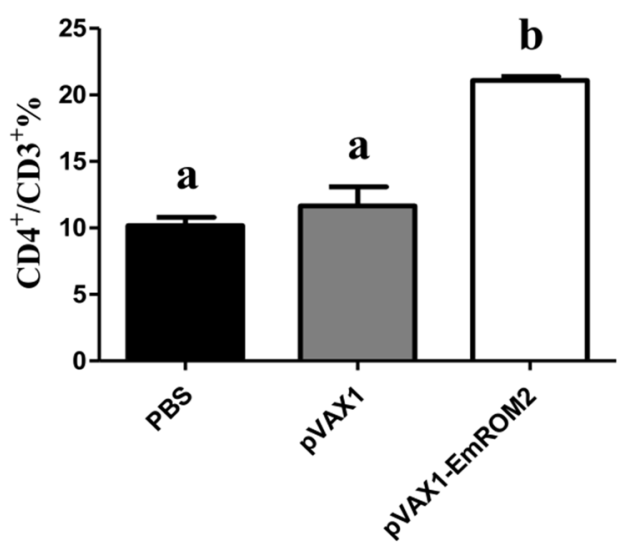

C

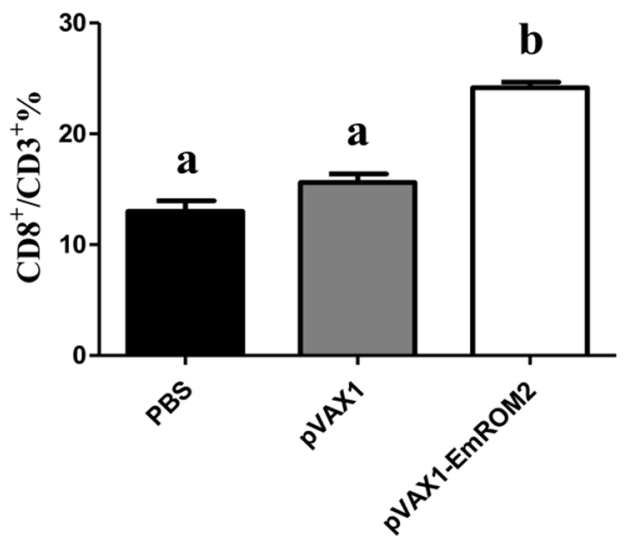

B

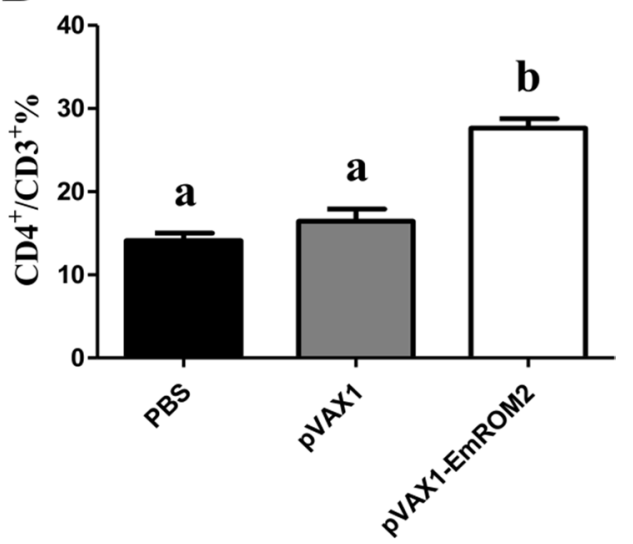

D

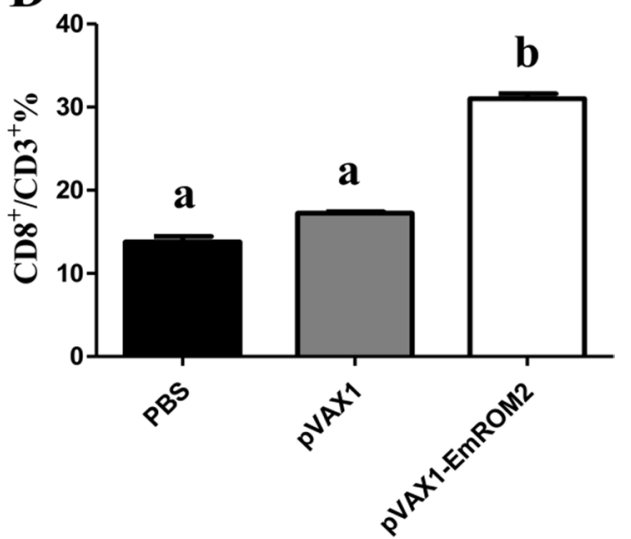

Figure 5 Changes of splenic T lymphocytes subsets induced by pVAX1-EmROM2 in chickens. A Proportion of CD4 $4^{+} / \mathrm{CD} 3^{+}$splenic T lymphocytes in chickens 1 week after the primary vaccination; B Proportion of $\mathrm{CD} 4^{+} / \mathrm{CD}^{+}{ }^{+}$splenic $T$ lymphocytes in chickens 1 week after the booster vaccination; $\mathbf{C}$ Proportion of $\mathrm{CD}^{+} / \mathrm{CD} 3^{+}$splenic T lymphocytes in chickens 1 week after the primary vaccination; $\mathbf{D}$ Proportion of $\mathrm{CD} 8^{+} /$ $\mathrm{CD}^{+}$splenic T lymphocytes in chickens 1 week after the booster vaccination. Significant difference $(p<0.05)$ between different groups was annotated with different letters. No significant difference $(p>0.05)$ between different groups was annotated with the same letter.

the invasion of apicomplexan protozoa, they were considered as new candidate antigens for developing newgeneration vaccines $[13,14]$. According to the previous study, chickens vaccinated with rETRHO1 (recombinant protein of E. tenella rhomboid-like protein) and pVAX1Rho (a DNA vaccine of E. tenella rhomboid-like protein) elicited humoral and cell-mediated immunity and generated protection against infection by E. tenella in chickens $[14,33]$. The present study was conducted to evaluate the immunogenicity and protective efficacy of EmROM2 in forms of recombinant protein (rEmROM2) and DNA (pVAX1-EmROM2), the results showed that EmROM2 activated notable humoral and cell-mediated immunity and provided partial protection against $E$. maxima. These results demonstrated that EmROM2 protein and DNA are effective vaccine candidates against E. maxima.
It has been reported that cell-mediated immunity plays a major role, and humoral immunity plays a minor role in the process of immunoprotection against coccidiosis [34-36]. In the present study, the proportion of $\mathrm{CD} 8^{+}$ and $\mathrm{CD} 4^{+} \mathrm{T}$ lymphocytes was obviously enhanced in the vaccinated chickens, indicating EmROM2 protein and DNA could induce cellular immune responses. Partially, cytokines could control and regulate the responses of $\mathrm{T}$ cells against coccidiosis. Th1-type cytokines (e.g. IFN- $\gamma$ and IL-2), relating to cellular immunity, are regarded to be predominant against coccidiosis [36, 37]. IFN- $\gamma$ are important in the process of regulating anticoccidial immune responses, because it can activate the phagocytosis of macrophages and killing effect of NK cells and CTLs [35]. IL-2 can induce the proliferation of T cells in vitro, and increase the proportion of $\mathrm{CD}^{+}$and $\mathrm{CD} 4^{+}$ 

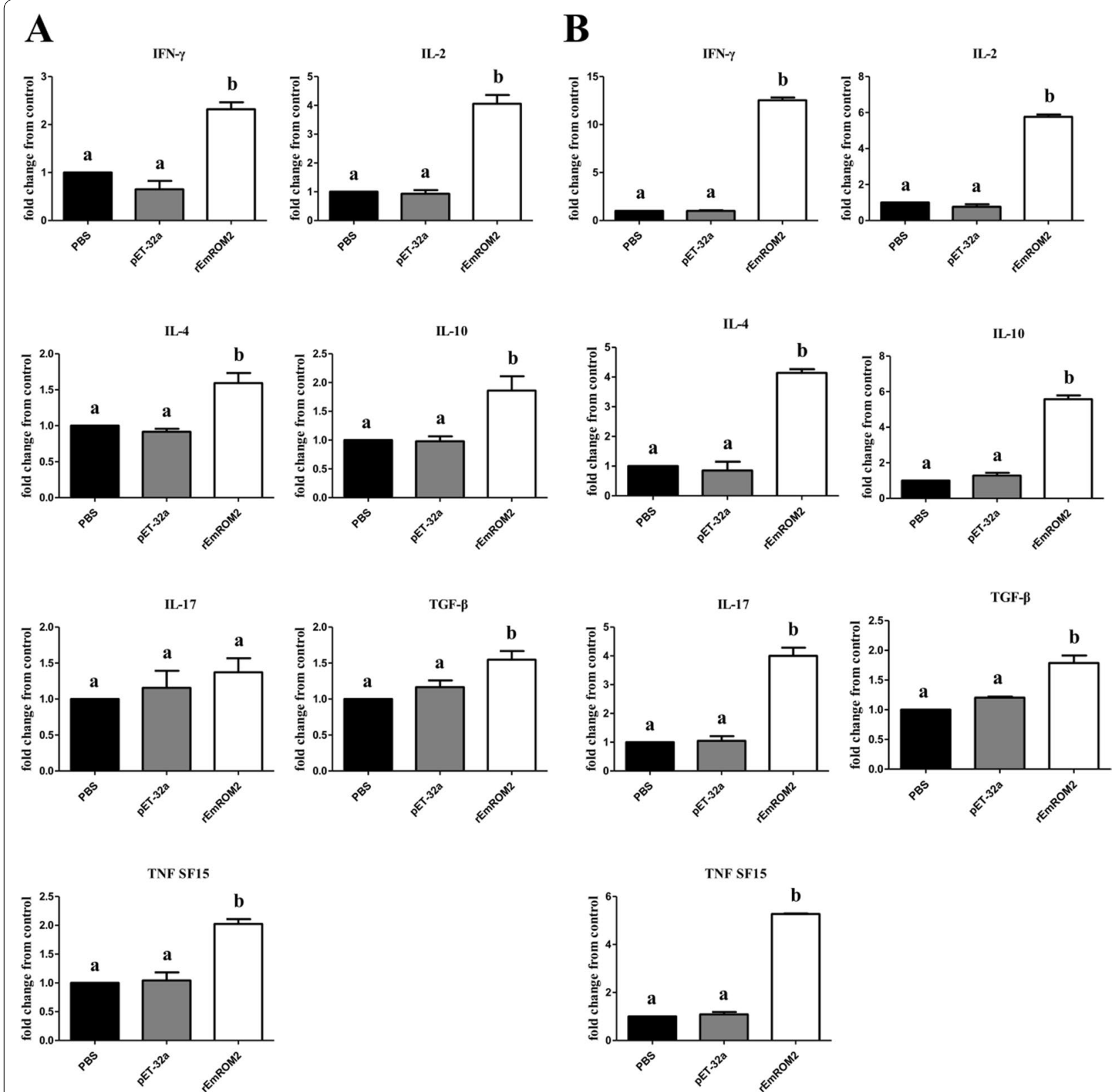

Figure 6 Changes of transcriptional level of cytokines in splenic lymphocytes induced by rEmROM2. A 1 week after the primary vaccination; $\mathbf{B} 1$ week after the booster vaccination. Significant difference $(p<0.05)$ between different groups was annotated with different letters. No significant difference $(p>0.05)$ between different groups was annotated with the same letter.

$\mathrm{T}$ lymphocytes in peripheral blood when co-delivered with vaccines in vivo [38]. In the present study, the transcriptional level of IFN- $\gamma$ and IL-2 in splenic lymphocytes from chickens vaccinated with rEmROM2 and pVAX1-EmROM2 were obviously upregulated compared with the control groups $(p<0.05)$. Similarly, the transcriptional level of other five major cytokines (IL-4, IL-10, IL-17, TGF- $\beta 4$ and TNF SF15) was significantly increased via the vaccination. The role of antibodies is controversial, and many reports suggest that antibodies contribute to, but are not fundamental function [39]. In some cases, however, antibodies seem to be involved in protection against coccidiosis [39, 40]. In this study, the level of specific IgG antibody was increased significantly in chickens vaccinated with rEmROM2 or pVAX1EmROM2. In short, the changes in cytokines and IgG 

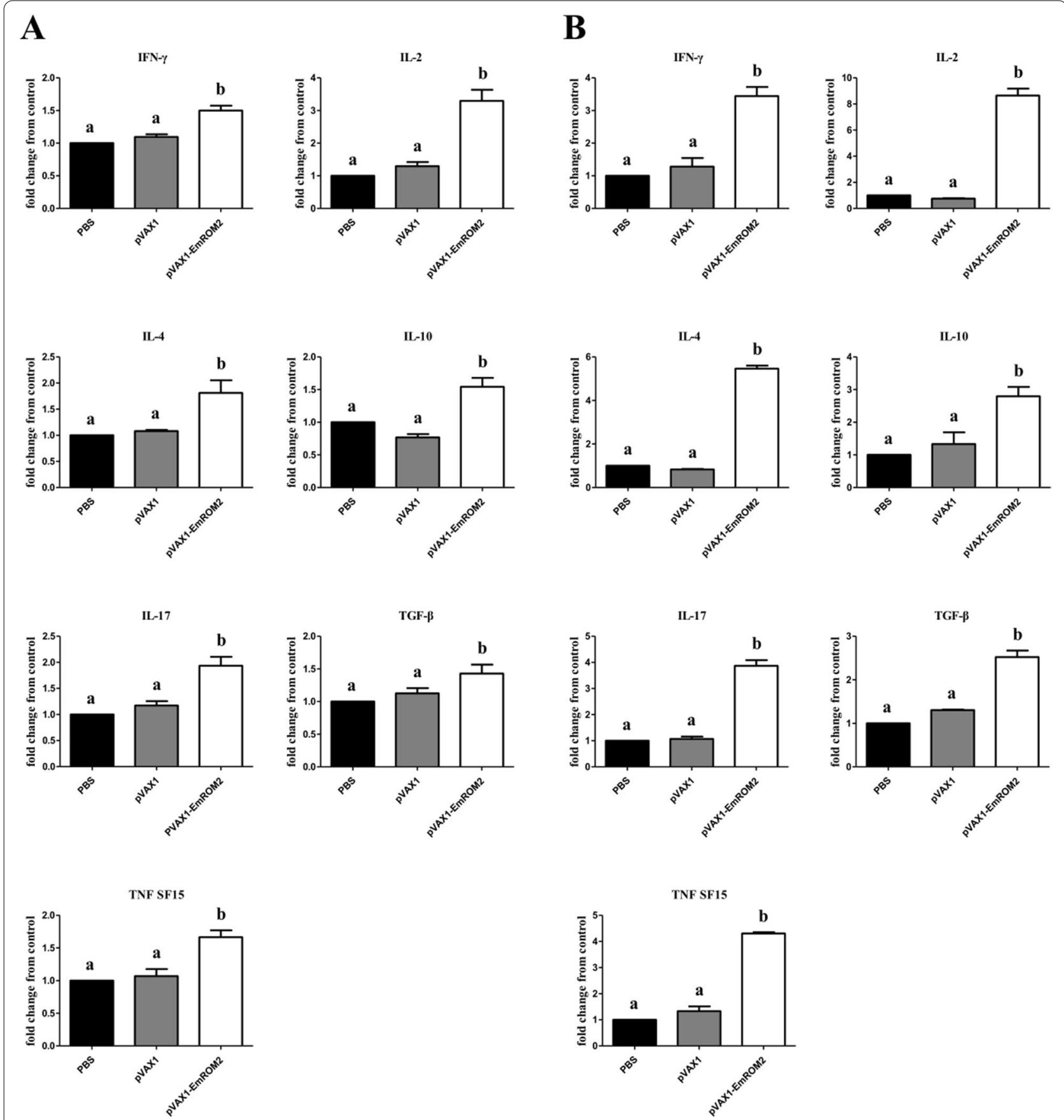

Figure 7 Changes of transcriptional level of cytokines in splenic lymphocytes induced by pVAX1-EmROM2. A 1 week after the primary vaccination; B 1 week after the booster vaccination. Significant difference $(p<0.05)$ between different groups was annotated with different letters. No significant difference $(p>0.05)$ between different groups was annotated with the same letter.

antibody revealed that rEmROM2 and pVAX1-EmROM2 could induce significant cellular and humoral immunity.

In this study, vaccination with EmROM2 protein or DNA significantly alleviated intestinal lesions, bodyweight loss, reduced oocyst output caused by E. maxima.
Moreover, it induced ACI values of more than 160 (170.60 and 162.74), showing partial protection against $E$. maxima infection. Furthermore, the protective efficacy could be enhanced by some methods. For example, some novel adjuvants including plant-derived adjuvants (such 

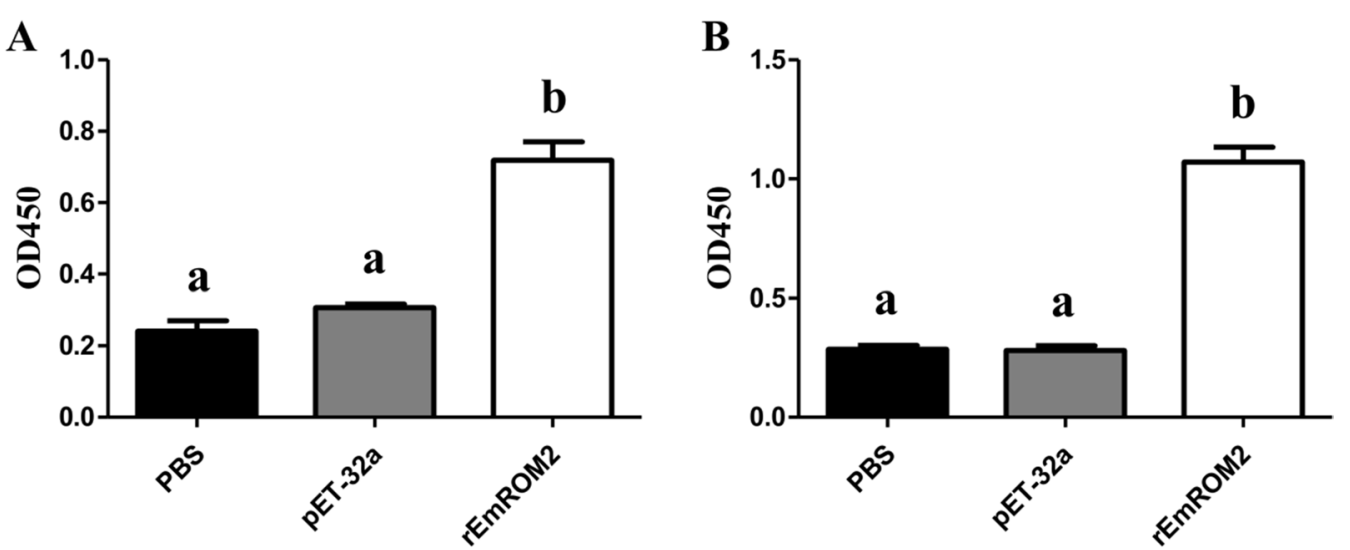

Figure 8 Changes of serum IgG level induced by rEmROM2 in chickens. A 1 week after the primary vaccination; B 1 week after the booster vaccination. Significant difference $(p<0.05)$ between different groups was annotated with different letters. No significant difference $(p>0.05)$ between different groups was annotated with the same letter.
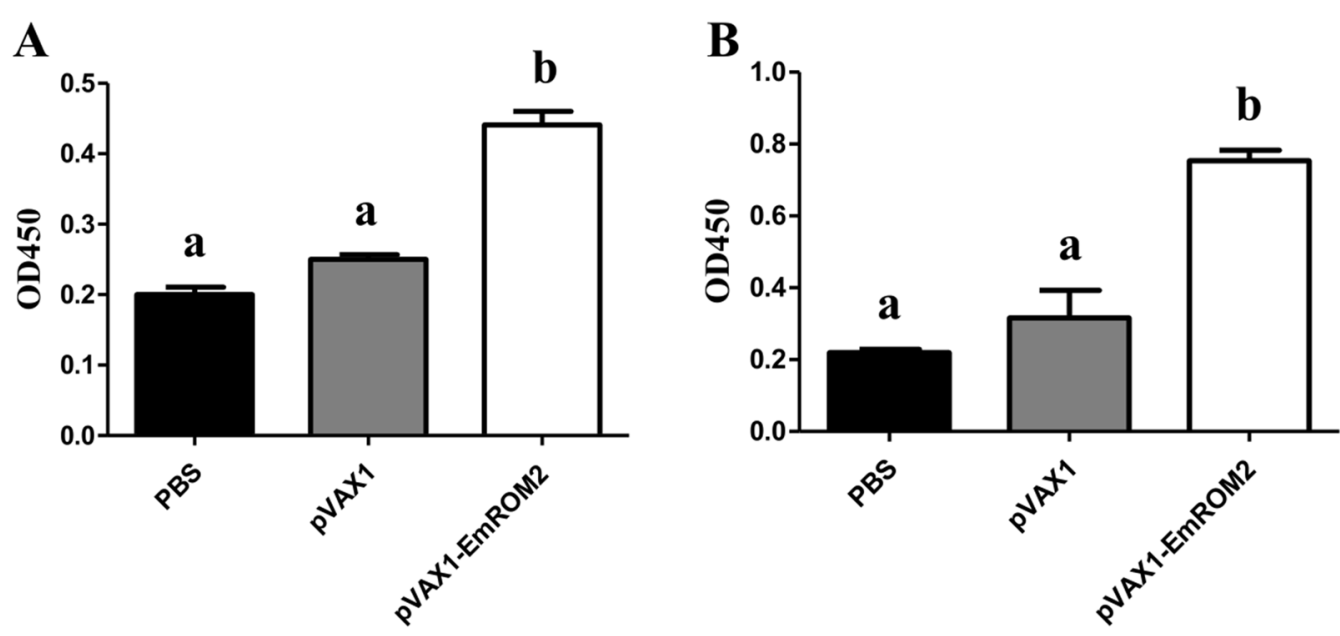

Figure 9 Changes of serum IgG level induced by pVAX1-EmROM2 in chickens. A 1 week after the primary vaccination; B 1 week after the booster vaccination. Significant difference $(p<0.05)$ between different groups was annotated with different letters. No significant difference $(p>0.05)$ between different groups was annotated with the same letter.

as saponins, and lectins) and nanoparticles (e.g. polymeric nanoparticles, inorganic nanoparticles and viruslike particles) were reported could activate or strengthen immune responses $[41,42]$. Therefore, protective efficacy induced by rEmROM2 and pVAX1-EmROM2 can be augmented through co-administered with these adjuvants. Additionally, the protective efficacy of vaccines could also be improved by optimizing the vaccination route, dose, time and age of primary vaccination in chickens [43].

\section{Supplementary Information}

The online version contains supplementary material available at https://doi. org/10.1186/s13567-020-00886-7.
Additional file 1. SDS-PAGE analysis of the expression of pET-

32a-EmROM2 in different time. Lane M: standard protein molecular weight marker; lane 1: pET-32a bacterial lysate; lane 2: pET-32a induced by IPTG for 5 h; lane 3-lane 8: pET-32a-EmROM2 induced by IPTG for 0-5 h.

\section{Abbreviations}

ACl: Anti-coccidial index; bp: Base pairs; BSA: Bovine serum albumin; cDNA: Complementary DNA; Ct: Cycle threshold; DAB: 3,3'-Diaminobenzidine tetrahydrochloride; EmROM2: Rhomboid protein 2 of Eimeria maxima; E. maxima: Eimeria maxima; E. tenella: Eimeria tenella; ELISA: Enzyme-linked immunosorbant assay; HRP: Horseradish peroxidase; IgG: Immunoglobulin G; kDa: Kilo dalton; M: Mole/L; mRNA: Message RNA; OD: Optical density; PBS: Phosphate buffered saline; PBST: Phosphate buffered saline-Tween; PCR: Polymerase chain reaction; qRT-PCR: Quantitative real-time PCR; RIPA: Radio immunoprecipitation assay; rEmROM2: EmROM2 recombinant protein; RT-PCR: Reverse transcription-PCR; SDS-PAGE: Sodium dodecyl sulfate polyacrylamide gel electrophoresis; TMB: 3,3,5,5'-Tetramethyl benzidine. 


\section{Acknowledgements}

We gratefully thank Siying Chen, Wenxi Ding, Xiaoting Sun, Mingyue Wang, Chen Chen, Xiaogian Liu and Junzhi Su for their contributions to the animal experiment.

\section{Authors' contributions}

XS conceived and designed the study. XL, RY and LX helped in the study design and analyzed the data. YC drafted the manuscript and SMA helped to revise the manuscript. DT contributed to the main experiment. YC performed the laboratory tests. All authors read and approved the final manuscript.

\section{Funding}

This work was supported by the National Natural Science Foundation of China (Grant No. 31672545, 31972705, and 31661143017), the National Key Research and Development Program of China (Grant No. 2017YFD0500400), and the Priority Academic Program Development of Jiangsu Higher Education Institutions (PAPD).

\section{Availability of data and materials}

The datasets during the current study available from the corresponding author on reasonable request.

\section{Ethics approval and consent to participate}

Animal experiments were approved by the Committee on Experimental Animal Welfare and Ethics of Nanjing Agricultural University (Approval number: PAT2020001).

\section{Consent for publication}

Not applicable.

\section{Competing interests}

The authors declare that they have no competing interests.

\section{Author details}

${ }^{1}$ MOE Joint International Research Laboratory of Animal Health and Food Safety, College of Veterinary Medicine, Nanjing Agricultural University, Nanjing 210095, China. ${ }^{2}$ Nanjing Ringpai Vet Hospital Co., Ltd., Nanjing 210012 , China. ${ }^{3}$ Department of Pathobiology, PMAS Arid Agriculture University, Rawalpindi 41000, Pakistan.

Received: 12 October 2020 Accepted: 23 December 2020 Published online: 18 February 2021

\section{References}

1. Blake DP, Tomley FM (2014) Securing poultry production from the everpresent Eimeria challenge. Trends Parasitol 30:12-19

2. Shirley MW, Smith AL, Blake DP (2007) Challenges in the successful control of the avian coccidia. Vaccine 25:5540-5547

3. Chapman HD, Jeffers TK (2014) Vaccination of chickens against coccidiosis ameliorates drug resistance in commercial poultry production. Int J Parasitol Drugs Drug Resist 4:214-217

4. Allen PC, Fetterer RH (2002) Recent advances in biology and immunobiology of Eimeria species and in diagnosis and control of infection with these coccidian parasites of poultry. Clin Microbiol Rev 15:58-65

5. Clarke L, Fodey TL, Crooks SR, Moloney M, O'Mahony J, Delahaut P, O'Kennedy R, Danaher M (2014) A review of coccidiostats and the analysis of their residues in meat and other food. Meat Sci 97:358-374

6. Meunier M, Chemaly M, Dory D (2016) DNA vaccination of poultry: the current status in 2015. Vaccine 34:202-211

7. Sharman PA, Smith NC, Wallach MG, Katrib M (2010) Chasing the golden egg: vaccination against poultry coccidiosis. Parasite Immunol 32:590-598

8. Tian L, Li W, Huang X, Tian D, Liu J, Yang X, Liu L, Yan R, Xu L, Li X, Song X (2017) Protective efficacy of coccidial common antigen glyceraldehyde 3-phosphate dehydrogenase (GAPDH) against challenge with three Eimeria SPECIES. Front Microbiol 8:1245

9. Li J, Zhang X, Liu Q, Yin J, Yang J (2006) Eimeria tenella: cloning of a novel Eimeria tenella cDNA encoding a protein related to rhomboid family from F2 hybrid strain. Exp Parasitol 113:215-220
10. Dowse TJ, Koussis K, Blackman MJ, Soldati-Favre D (2008) Roles of proteases during invasion and egress by Plasmodium and Toxoplasma. Subcell Biochem 47:121-139

11. Santos J, Graindorge A, Soldati-Favre D (2012) New insights into parasite rhomboid proteases. Mol Biochem Parasitol 182:27-36

12. Shen B, Buguliskis JS, Lee TD, Sibley LD (2014) Functional analysis of rhomboid proteases during Toxoplasma invasion. mBio 5:e01795-01714

13. Foroutan M, Zaki L, Tavakoli S, Soltani S, Taghipour A, Ghaffarifar F (2019) Rhomboid antigens are promising targets in the vaccine development against Toxoplasma gondii. EXCLI J 18:259-272

14. Li J, Zheng J, Gong P, Zhang X (2012) Efficacy of Eimeria tenella rhomboidlike protein as a subunit vaccine in protective immunity against homologous challenge. Parasitol Res 110:1139-1145

15. Baker RP, Wijetilaka R, Urban S (2006) Two Plasmodium rhomboid proteases preferentially cleave different adhesins implicated in all invasive stages of malaria. PLoS Pathog 2:e113

16. Dowse TJ, Soldati D (2005) Rhomboid-like proteins in Apicomplexa: phylogeny and nomenclature. Trends Parasitol 21:254-258

17. Yang G, Li J, Zhang X, Zhao Q, Liu Q, Gong P (2008) Eimeria tenella: construction of a recombinant fowlpox virus expressing rhomboid gene and its protective efficacy against homologous infection. Exp Parasitol 119:30-36

18. Wang Q, Li J, Zhang X, Liu Q, Liu C, Ma G, Cao L, Gong P, Cai Y, Zhang G (2009) Protective immunity of recombinant Mycobacterium bovis BCG expressing rhomboid gene against Eimeria tenella challenge. Vet Parasitol 160:198-203

19. Tomley $F$ (1997) Techniques for isolation and characterization of apical organelles from Eimeria tenella sporozoites. Methods 13:171-176

20. Song X, Zhao X, Xu L, Yan R, Li X (2017) Immune protection duration and efficacy stability of DNA vaccine encoding Eimeria tenella TA4 and chicken IL-2 against coccidiosis. Res Vet Sci 111:31-35

21. Sasai K, Aita M, Lillehoj HS, Miyamoto T, Fukata T, Baba E (2000) Dynamics of lymphocyte subpopulation changes in the cecal tonsils of chickens infected with Salmonella enteritidis. Vet Microbiol 74:345-351

22. Song H, Song X, Xu L, Yan R, Shah MA, Li X (2010) Changes of cytokines and IgG antibody in chickens vaccinated with DNA vaccines encoding Eimeria acervulina lactate dehydrogenase. Vet Parasitol 173:219-227

23. Livak KJ, Schmittgen TD (2001) Analysis of relative gene expression data using real-time quantitative PCR and the 2(-Delta Delta C(T)) Method. Methods 25:402-408

24. Holdsworth PA, Conway DP, McKenzie ME, Dayton AD, Chapman HD, Mathis GF, Skinner JT, Mundt HC, Williams RB, World Association for the Advancement of Veterinary P (2004) World Association for the Advancement of Veterinary Parasitology (WAAVP) guidelines for evaluating the efficacy of anticoccidial drugs in chickens and turkeys. Vet Parasitol 121:189-212

25. McManus EC, Campbell WC, Cuckler AC (1968) Development of resistance to quinoline coccidiostats under field and laboratory conditions. J Parasitol 54:1190-1193

26. Johnson J, Reid WM (1970) Anticoccidial drugs: lesion scoring techniques in battery and floor-pen experiments with chickens. Exp Parasitol 28:30-36

27. Morehouse NF, Baron RR (1970) Coccidiosis: evaluation of coccidiostats by mortality, weight gains, and fecal scores. Exp Parasitol 28:25-29

28. Chapman HD, Shirley MW (1989) Sensitivity of field isolates of Eimeria species to monensin and lasalocid in the chicken. Res Vet Sci 46:114-117

29. Belli SI, Mai K, Skene CD, Gleeson MT, Witcombe DM, Katrib M, Finger A, Wallach MG, Smith NC (2004) Characterisation of the antigenic and immunogenic properties of bacterially expressed, sexual stage antigens of the coccidian parasite Eimeriamaxima. Vaccine 22:4316-4325

30. Huang J, Zhang Z, Li M, Song X, Yan R, Xu L, Li X (2015) Eimeria maxima microneme protein 2 delivered as DNA vaccine and recombinant protein induces immunity against experimental homogenous challenge. Parasitol Int 64:408-416

31. Huang J, Zhang Z, Li M, Song X, Yan R, Xu L, Li X (2015) Immune protection of microneme 7 (EmMIC7) against Eimeria maxima challenge in chickens. Avian Pathol 44:392-400

32. Liu J, Liu L, Li L, Tian D, Li W, Xu L, Yan R, Li X, Song X (2018) Protective immunity induced by Eimeria common antigen 14-3-3 against Eimeria tenella, Eimeria acervulina and Eimeria maxima. BMC Vet Res 14:337 
33. Liu Y, Zheng J, Li J, Gong P, Zhang X (2013) Protective immunity induced by a DNA vaccine encoding Eimeria tenella rhomboid against homologous challenge. Parasitol Res 112:251-257

34. Rose ME, Hesketh P (1979) Immunity to coccidiosis: T-lymphocyte- or B-lymphocyte-deficient animals. Infect Immun 26:630-637

35. Lillehoj HS (1998) Role of T lymphocytes and cytokines in coccidiosis. Int J Parasitol 28:1071-1081

36. Cornelissen JB, Swinkels WJ, Boersma WA, Rebel JM (2009) Host response to simultaneous infections with Eimeria acervulina, maxima and tenella: a cumulation of single responses. Vet Parasitol 162:58-66

37. Shah MA, Song X, Xu L, Yan R, Li X (2011) Construction of DNA vaccines encoding Eimeria acervulina cSZ-2 with chicken IL-2 and IFN-gamma and their efficacy against poultry coccidiosis. Res Vet Sci 90:72-77

38. Hilton LS, Bean AG, Lowenthal JW (2002) The emerging role of avian cytokines as immunotherapeutics and vaccine adjuvants. Vet Immunol Immunopathol 85:119-128

39. Constantinoiu CC, Molloy JB, Jorgensen WK, Coleman GT (2008) Antibody response against endogenous stages of an attenuated strain of Eimeria tenella. Vet Parasitol 154:193-204
40. Dalloul RA, Lillehoj HS (2006) Poultry coccidiosis: recent advancements in control measures and vaccine development. Expert Rev Vaccines 5:143-163

41. Sander VA, Corigliano MG, Clemente M (2019) Promising plant-derived adjuvants in the development of coccidial vaccines. Front Vet Sci 6:20

42. Zhao L, Seth A, Wibowo N, Zhao CX, Mitter N, Yu C, Middelberg AP (2014) Nanoparticle vaccines. Vaccine 32:327-337

43. Song X, Xu L, Yan R, Huang X, Shah MA, Li X (2009) The optimal immunization procedure of DNA vaccine pCDNA-TA4-IL-2 of Eimeria tenella and its cross-immunity to Eimeria necatrix and Eimeria acervulina. Vet Parasitol 159:30-36

\section{Publisher's Note}

Springer Nature remains neutral with regard to jurisdictional claims in published maps and institutional affiliations.
Ready to submit your research? Choose BMC and benefit from:

- fast, convenient online submission

- thorough peer review by experienced researchers in your field

- rapid publication on acceptance

- support for research data, including large and complex data types

- gold Open Access which fosters wider collaboration and increased citations

- maximum visibility for your research: over 100M website views per year

At BMC, research is always in progress.

Learn more biomedcentral.com/submissions 